\title{
On the Optimal Policy Structure in Serial Inventory Systems with Lost Sales
}

\author{
Woonghee Tim Huh, Columbia University \\ Ganesh Janakiraman† New York University
}

May 21, 2008

Revised: July 30, 2008 and December 21, 2008

\begin{abstract}
We study a periodically reviewed, serial inventory system in which excess demand from external customers is lost. We derive elementary properties of the vector of optimal order quantities in this system. In particular, we derive bounds on the sensitivity (or, more mathematically, the derivative) of the optimal order quantity at each stage to the vector of the current inventory levels. Our analysis uses the concept of $L$-naturalconvexity, which was studied in discrete convex analysis (Murota (2003)) and recently used by Zipkin (2008) for studying single-stage inventory systems with lost sales. We also remark on how our analysis extends to models with capacity constraints and/or backordering.
\end{abstract}

${ }^{*}$ Industrial Engineering and Operations Research Department, Columbia University, 500 West 120th Street, New York, NY 10027, USA. Email: huh@ieor.columbia.edu. Research supported partially by NSF grant DMS-0732169.

†Stern School of Business, New York University, 44 West 4th Street, New York, NY 10012, USA. Email: gjanakir@stern.nyu. edu. 


\section{Introduction}

We consider a periodically-reviewed, single-item inventory system with $J$ stages in series. Excess demand that cannot be satisfied immediately at the most downstream (i.e., customerfacing) stage is lost. Lost sales inventory systems are known to be difficult to analyze, and the optimal policy for even the single-stage system is complicated when the order delivery lead-time is positive. (When the lead-time is zero, the problem becomes the standard multiperiod newsvendor problem for which order-up-to policies are optimal.) For single-stage systems, a partial characterization of the structure of the optimal policy has been provided by Karlin and Scarf (1959) and Morton (1969), and their results are recently reinterpreted and strengthened by Zipkin (2008). For multi-echelon systems, no such results are known; in fact, to our knowledge, this work is the first attempt to study the structure of optimal policies in multi-echelon systems with lost sales. In particular, we provide bounds on the sensitivity of the optimal order quantity at each stage with respect to the vector of the inventory levels.

In this paper, we use the concept of $L$-natural-convexity ( $L^{\natural}$-convexity) from discrete convex analysis (Murota (2003)), recently used by Zipkin (2008) for single stage systems, to establish properties of the optimal policy in serial systems. Another recent example of its use in inventory theory is Lu and Song (2005). We refer the reader to Chapter VII of Fujishige (2005), Murota (2003), and Zipkin (2008) for a more detailed discussion on this concept.

Our analysis is based on two main ideas. The first one is to consider $J$ equivalent versions, one for each stage, of the stochastic dynamic program corresponding to the problem of minimizing the expected costs incurred by this system over some finite horizon of periods. The $j^{\text {th }}$ version is used to generate the sensitivity result related to the order quantity of stage $j$. The second one is to transform the state vector such that a state can be represented by a so-called "bi-echelon inventory vector", with respect to which the $L^{\natural}$-convexity property can be established. To the best of our knowledge, both of these ideas are novel in the inventory literature. 


\section{Model and Result}

We consider a serial inventory system with lost sales under periodic review. The system consists of $J \geq 1$ stages, indexed by $j=0,1, \ldots, J-1$. The lowest stage facing exogenous demand is represented by stage 0 , stage $j$ orders from stage $j+1$ where $j \in\{0, \ldots, J-2\}$, and stage $J-1$ orders from an outside supplier with infinite supply. The replenishment leadtime from one stage to another is deterministic, and we assume initially that each lead-time is 1 period. (In Section 4, we comment on how our analysis extends to the case of arbitrary lead times.) In each period, the following sequence of events occurs: (1) receipt of delivery at every stage, (2) order placement at every stage, and (3) demand realization. The demand in each period is satisfied to the extent possible, and any demand that cannot be satisfied immediately is lost. Let $p \geq 0$ represent the per-unit lost sales penalty cost, and let $H^{j} \geq 0$ denote the holding cost at stage $j$, that is, the cost for holding one unit at stage $j$ for a period. These costs are charged at the end of a period. For any $j \in\{0,1, \ldots, J-1\}$, let $x^{j} \geq 0$ denote the stage- $j$ inventory level after receiving deliveries at the beginning of a period, and let $\tilde{q}^{j} \in\left[0, x^{j+1}\right]$, where $x^{J}$ is taken to be $\infty$, denote the quantity ordered by stage $j$ in a period. Thus, the vector $\left(x^{0}, x^{1}, \ldots, x^{J-1}\right)$ represents the state at the beginning of a period after receiving deliveries, and the vector $\left(\tilde{q}^{0}, \tilde{q}^{1}, \ldots, \tilde{q}^{J-1}\right)$ represents the action in a period. We use $\mathbf{x}$ and $\tilde{\mathbf{q}}$ to denote the vectors $\left(x^{0}, \ldots, x^{J-1}\right)$, and $\left(\tilde{q}^{0}, \ldots, \tilde{q}^{J-1}\right)$, respectively. Let $D_{t}$ denote the demand in period $t$. We assume that demands are independently distributed across periods.

Next, we define the dynamic program for this system for a planning horizon of $T$ periods indexed by $t=1,2, \ldots, T$. Periods are indexed forwards, i.e., period $t+1$ follows period $t$. Given a state-action pair of $\mathbf{x}$ and $\tilde{\mathbf{q}}$ in period $t$, the stage- $j$ inventory level in period $t+1$ is given by

$$
\begin{cases}\left(x^{0}-D_{t}\right)^{+}+\tilde{q}^{0} & \text { if } j=0 \\ x^{j}-\tilde{q}^{j-1}+\tilde{q}^{j} & \text { if } j \in\{1, \ldots, J-1\} .\end{cases}
$$


Let $\tilde{f}_{T+1}(\mathbf{x})=0$ for all $\mathbf{x}$. Consider any $t \in\{1, \ldots, T\}$. Let $\gamma \in(0,1]$ denote the discount factor for capturing the time value of money. Define

$$
\begin{aligned}
\tilde{f}_{t}(\mathbf{x})= & E\left[H^{0} \cdot\left(x^{0}-D_{t}\right)^{+}+\sum_{j=1}^{J-1} H^{j} \cdot x^{j}+p \cdot\left(D_{t}-x^{0}\right)^{+}\right] \\
+ & \gamma \cdot \min _{\tilde{\mathbf{q}}} E\left[\tilde{f}_{t+1}\left(\left(x^{0}-D_{t}\right)^{+}+\tilde{q}^{0}, x^{1}-\tilde{q}^{0}+\tilde{q}^{1}, \ldots, x^{J-1}-\tilde{q}^{J-2}+\tilde{q}^{J-1}\right)\right] \\
& \text { s. t. } \quad 0 \leq \tilde{q}^{j} \leq x^{j+1}, j=0, \ldots, J-1 .
\end{aligned}
$$

Let $\tilde{\mathbf{q}}_{t}^{*}(\mathbf{x})$ denote a minimizing vector, $\tilde{\mathbf{q}}$, in the optimization problem above. Thus, $\tilde{q}_{t}^{j *}$ denotes the order quantity for stage $j$, according to this vector. [Note: When the optimization problem above does not have a unique solution, any statements we make on properties of $\tilde{\mathbf{q}}_{t}^{*}$ should be taken to mean the existence of an optimal selector $\tilde{\mathbf{q}}_{t}^{*}(\mathbf{x})$ (precisely defined in Section 3.4) with those properties.]

We are now ready to state the main result of the paper.

Theorem 1. Assume that $\tilde{\mathbf{q}}_{t}^{*}$ is a differentiable function of $\mathbf{x}$. Then, the following inequalities hold:

$$
\begin{gathered}
-1 \leq \frac{\partial \tilde{q}_{t}^{k *}}{\partial x^{k}} \leq \cdots \leq \frac{\partial \tilde{q}_{t}^{k *}}{\partial x^{0}} \leq 0 \text { for every } k \in\{0, \ldots, J-1\} \\
0 \leq \frac{\partial \tilde{q}_{t}^{k *}}{\partial x^{J-1}} \leq \cdots \leq \frac{\partial \tilde{q}_{t}^{k *}}{\partial x^{k+1}} \leq 1 \text { for every } k \in\{0, \ldots, J-2\}, \text { and } \\
\frac{\partial \tilde{q}_{t}^{k *}}{\partial x^{k+1}}-\frac{\partial \tilde{q}_{t}^{k *}}{\partial x^{k}} \leq 1 \text { for every } k \in\{0, \ldots, J-2\}
\end{gathered}
$$

If $\tilde{\mathbf{q}}_{t}^{*}$ is not differentiable, then the inequalities above hold after replacing every quantity of the form $\frac{\partial \tilde{q}_{t}^{k *}}{\partial x^{j}}$ with $\frac{\tilde{q}_{t}^{k *}\left(\mathbf{x}+\boldsymbol{\epsilon}^{j}\right)-\tilde{q}_{t}^{k *}(\mathbf{x})}{\epsilon}$, where $\boldsymbol{\epsilon}^{j}$ is a vector with $\epsilon$ in its $j^{\text {th }}$ component and zero in all the other components and $\epsilon$ is any strictly positive number.

In words, Theorem 1 states that the optimal order quantity at stage $k$ is a decreasing function of the inventory at any downstream stage $j$ (i.e., $j \leq k$ ), and that the rate of this decrease is smaller than 1 . On the other hand, the optimal order quantity at stage $k$ is an increasing function of the inventory at any upstream stage $j$ (i.e., $j>k$ ), and the rate of this increase is also smaller than 1. Furthermore, the sensitivity of the optimal order quantity at a stage to the inventory level at a downstream (upstream) stage is greater the 
less downstream (upstream) the latter stage is relative to the former stage. Finally, the rate of decrease of the optimal order quantity at a stage with respect to any downstream stage's inventory plus the rate of its increase with respect to any upstream stage's inventory is itself smaller than 1.

We present the proof of this theorem in Section 3.

\section{Proof of Theorem 1}

\subsection{Preliminary Results}

Let $V \subseteq \Re^{n}$ be a polyhedron that forms a lattice. A function $f: V \rightarrow \Re$ is submodular provided that

$$
f(\mathbf{x})+f(\mathbf{y}) \geq f(\mathbf{x} \vee \mathbf{y})+f(\mathbf{x} \wedge \mathbf{y})
$$

for any pair of $\mathbf{x}$ and $\mathbf{y}$ in $V$, where $\vee$ and $\wedge$ are the component-wise minimum and component-wise maximum operators. Let $\mathbf{e}=(1,1, \ldots, 1)$ be the vector of 1 's with an appropriate length. Following Zipkin (2008), we say that $f: V \rightarrow \Re$ is $L^{\natural}$-convex if

$$
\psi(\mathbf{v}, \zeta)=f(\mathbf{v}-\zeta \mathbf{e}), \quad \zeta \leq 0
$$

is submodular on $\left\{(\mathbf{v}, \zeta) \mid \mathbf{v} \in V, \zeta \in \Re^{-}, \mathbf{v}-\zeta \mathbf{e} \in V\right\}$. The following basic properties of $L^{\natural}$-convexity are straightforward extensions of results presented in Zipkin's paper.

\section{Lemma 2.}

(a) If $f(\mathbf{v})$ is $L^{\natural}$-convex, then $\psi(\mathbf{v}, \zeta)=f(\mathbf{v}-\zeta \mathbf{e})$ is also $L^{\natural}$-convex.

(b) Let $r^{j}$ and $u^{j}$ be fixed for all $j \in\{1, \ldots, m\}$ such that $r^{j} \leq u^{j}$, and let $\xi^{i j}$ and $\varsigma^{i j}$ be fixed for all $(i, j) \in\{1, \ldots, n\} \times\{1, \ldots, m\}$ such that $\varsigma^{i j} \leq \xi^{i j}$. Define

$$
\mathcal{S}=\left\{(v, w) \in \Re^{n} \times \Re^{m} \mid \varsigma^{i, j} \leq v^{i}-w^{j} \leq \xi^{i, j} \forall(i, j), \text { and } r^{j} \leq w^{j} \leq u^{j} \forall j\right\} .
$$

Suppose that $g(\mathbf{v}, \mathbf{w})$ is a $L^{\natural}$-convex function defined on $\mathcal{S}$. Let

$$
f(\mathbf{v})=\min _{\mathbf{w}}\{g(\mathbf{v}, \mathbf{w}) \mid(\mathbf{v}, \mathbf{w}) \in \mathcal{S}\}
$$

Then, $f$ is $L^{\natural}$-convex. 
(c) Let $\mathcal{I}$ be a subset of $\{1, \ldots, n\}$, and suppose that $g(\mathbf{v}, \zeta)$ is $L^{\natural}$-convex, defined on $\left\{\mathbf{v}: v^{i} \leq 0\right.$ for all $\left.i \in \mathcal{I}\right\}$. Let $\zeta(\mathbf{v})$ denote the largest value of $\zeta$ that solves

$$
\min _{\zeta}\left\{g(\mathbf{v}, \zeta) \mid v^{i} \leq \zeta \leq 0 \quad \text { for } i \in \mathcal{I}\right\}
$$

Then, $\zeta(\mathbf{v})$ is nondecreasing in $\mathbf{v}$, and $\zeta(\mathbf{v}+\omega \mathbf{e}) \leq \zeta(\mathbf{v})+\omega$ for $\omega>0$.

Proof. While part (a) is due to Zipkin (2008), parts (b) and (c) require minor modifications. We will first prove part (b), which slightly generalizes Lemma 2 of Zipkin (2008). Let

$$
\begin{aligned}
\psi(\mathbf{v}, \zeta) & =f(\mathbf{v}-\zeta \mathbf{e}) \\
& =\min _{\mathbf{w}}\left\{g(\mathbf{v}-\zeta \mathbf{e}, \mathbf{w}) \mid \varsigma^{i, j} \leq v^{i}-\zeta-w^{j} \leq \xi^{i, j} \forall(i, j), r^{j} \leq w^{i} \leq u^{j} \forall j\right\} \\
& =\min _{\tilde{\mathbf{w}}}\left\{g(\mathbf{v}-\zeta \mathbf{e}, \tilde{\mathbf{w}}-\zeta \mathbf{e}) \mid \varsigma^{i, j} \leq v^{i}-\tilde{w}^{j} \leq \xi^{i, j} \forall(i, j), r^{j} \leq \tilde{w}^{i}-\zeta \leq u^{j} \forall j\right\} .
\end{aligned}
$$

Since each constraint in the above minimization problem has exactly two non-zero variables of $(\mathbf{v}, \tilde{\mathbf{w}}, \zeta)$ and these two variables have the opposite signs, the feasible region forms a lattice (Topkis (1998), Example 2.2.7). Thus, $\psi(\mathbf{v}, \zeta)$ is submodular (Topkis (1998), Theorem 2.7.6), and we conclude that $f$ is $L^{\natural}$-convex.

We will now prove part (c). Since $g$ is submodular and the feasible region of the minimization problem forms a lattice, $\zeta(\mathbf{v})$ is nondecreasing in $\mathbf{v}$ by Theorem 2.8.2 of Topkis (1998). Also, for any $\omega>0$ and $\zeta \leq 0$ satisfying $\zeta>\zeta(\mathbf{v})+\omega$,

$$
g(\mathbf{v}+\omega \mathbf{e}, \zeta)-g(\mathbf{v}+\omega \mathbf{e}, \zeta(\mathbf{v})+\omega) \geq g(\mathbf{v}, \zeta-\omega)-g(\mathbf{v}, \zeta(\mathbf{v}))>0 .
$$

The first inequality in (1) is true because of the following argument. ${ }^{1}$ Define

$$
\psi\left(\mathbf{v}, \zeta, \zeta^{\prime}\right)=g\left(\mathbf{v}-\zeta^{\prime} \mathbf{e}, \zeta-\zeta^{\prime}\right)
$$

Since $g$ is $L^{\natural}$ convex, we know that $\psi$ is submodular in $\left(\mathbf{v}, \zeta, \zeta^{\prime}\right)$; in particular, for a given $\mathbf{v}$, $\psi\left(\mathbf{v}, \zeta, \zeta^{\prime}\right)$ is submodular in $\left(\zeta, \zeta^{\prime}\right)$. This implies that

$$
\psi(\mathbf{v}, \zeta-\omega,-\omega)-\psi(\mathbf{v}, \zeta(\mathbf{v}),-\omega) \geq \psi(\mathbf{v}, \zeta-\omega, 0)-\psi(\mathbf{v}, \zeta(\mathbf{v}), 0) .
$$

\footnotetext{
${ }^{1}$ An alternate proof of this inequality is based on the following property known as translationsubmodularity (see Theorems 7.2 and 7.29 of Fujishige (2005) and the paper by Murota and Shioura (2004)): if $g$ is $L^{\natural}$ convex, then $g(\mathbf{p})+g(\mathbf{q}) \geq g((\mathbf{p}-\alpha \cdot \mathbf{e}) \wedge \mathbf{q})+g(\mathbf{p} \vee(\mathbf{q}+\alpha \cdot \mathbf{e}))$ for all $\alpha \geq 0$. The desired inequality follows by choosing $\mathbf{p}=(\mathbf{v}+\omega \mathbf{e}, \zeta), \mathbf{q}=(\mathbf{v}, \zeta(\mathbf{v}))$ and $\alpha=\omega$ and from the facts that $(\mathbf{p}-\alpha \cdot \mathbf{e})=(\mathbf{v}, \zeta-\omega) \geq \mathbf{q}$ and $(\mathbf{q}+\alpha \cdot \mathbf{e})=(\mathbf{v}+\omega \mathbf{e}, \zeta(\mathbf{v})+\omega) \leq \mathbf{p}$.
} 
Observe that the left and right sides of the inequality above are equal to the left and middle expressions of (1), respectively. This completes the proof of the first inequality in (1).

The second inequality in (1) follows from the assumption that $\zeta-\omega>\zeta(\mathbf{v})$ and the fact that, for every $\zeta^{\prime \prime}>\zeta(\mathbf{v})$, the inequality $g\left(\mathbf{v}, \zeta^{\prime \prime}\right)>g(\mathbf{v}, \zeta(\mathbf{v}))$ holds due to the definition of $\zeta(\mathbf{v})$. Now, from (1), we obtain $g(\mathbf{v}+\omega \mathbf{e}, \zeta)>g(\mathbf{v}+\omega \mathbf{e}, \zeta(\mathbf{v})+\omega)$, which implies that $\zeta$ cannot be optimal for $\mathbf{v}+\omega \mathbf{e}$. Thus, $\zeta(\mathbf{v}+\omega \mathbf{e}) \leq \zeta(\mathbf{v})+\omega$.

\subsection{State Transformation}

We fix $k \in\{0 \ldots, J-1\}$, one of the stages of the system, whose optimal order quantity we will analyze, and introduce an alternate representation of the state. Define

$$
\begin{array}{ll}
w^{j}=x^{j}+x^{j+1}+\cdots+x^{k} & \text { for } j \in\{0,1, \ldots, k\}, \text { and, } \\
u^{j}=-\left(x^{k+1}+x^{k+2}+\ldots+x^{j}\right) & \text { for } j \in\{k+1, \ldots, J-1\} \text { and } k \leq J-2 .
\end{array}
$$

Let $\mathbf{w}=\left(w^{0}, \ldots, w^{k}\right)$. For $k \leq J-2$, let $\mathbf{u}=\left(u^{k+1}, \ldots, u^{J-1}\right)$. (For conciseness, we refrain from stating $k \leq J-2$ each time $\mathbf{u}$ is used. If $k=J-1$, $\mathbf{u}$ should be treated as the null vector.) We refer to $(\mathbf{w}, \mathbf{u})$ as the bi-echelon inventory vector anchored at $k$. Then, $(\mathbf{w}, \mathbf{u})$ is a valid representation of the state, where the corresponding state space is given by

$$
V=\left\{(\mathbf{w}, \mathbf{u}) \mid w^{0} \geq \cdots \geq w^{k} \geq 0 \geq u^{k+1} \geq \ldots \geq u^{J-1}\right\}
$$

Notice that $V$ is a lattice.

For our analysis, it is convenient to consider the following modifications to our model. First, we divide the set of ordering decisions into two steps, the first one for stage $k$ and the second one for all the other stages. Second, we no longer insist that the manager must satisfy demand to the maximum extent possible; we give the manager the flexibility of selecting the sales quantity (provided that the sales quantity does not exceed the demand and does not exceed the available inventory at stage 0). It is easy to show that it is optimal to satisfy demand to the maximum extent possible. Of these two modifications, the first is a technique which is new to the multi-echelon inventory literature whereas the second appears in Zipkin (2008). Now, the revised sequence of events is as follows: (1) receipt of delivery at all stages, 
(2a) order placement for stage $k,(2 \mathrm{~b})$ order placement for all the other stages, (3) demand realization, and (4) the sales quantity decision. We emphasize that the modified problem remains equivalent to the original problem, and these modifications are introduced for the convenience of our analysis only.

Let $q^{j} \geq 0$ denote the quantity ordered by stage $j$. For $j<J-1$, this quantity $q^{j}$ is constrained above by the amount of available inventory in the immediately upstream stage, $x^{j+1}$. Define

$$
\begin{array}{ll}
w_{+}^{j}=w^{j}-q^{j-1} & \text { for } j \in\{1, \ldots, k\}, \quad \text { and } \\
u_{+}^{j}=u^{j}-q^{j} & \text { for } j \in\{k+1, k+2, \ldots, J-1\} .
\end{array}
$$

Let $\hat{\mathbf{w}}_{+}=\left(w_{+}^{1}, \ldots, w_{+}^{k}\right)$ and $\mathbf{u}_{+}=\left(u_{+}^{k+1}, \ldots, u_{+}^{J-1}\right)$. Then, $\left(w^{0}, \hat{\mathbf{w}}_{+}, \mathbf{u}_{+}\right)$corresponds to the state in the next period, assuming that stage $k$ does not order in the current period, and the realized demand is zero. Further, let $\zeta \leq 0$ denote the negative of the order quantity of stage $k$ in the current period, i.e., $\zeta=-q^{k}$. It follows that $\left(\zeta, \hat{\mathbf{w}}_{+}, \mathbf{u}_{+}\right)$could be used to represent the order placement action in the current period; that is, given $(\mathbf{w}, \mathbf{u})$ and $\left(\zeta, \hat{\mathbf{w}}_{+}, \mathbf{u}_{+}\right)$, the vector of order quantities at all stages is completely determined. The feasible order placement action $\left(\zeta, \hat{\mathbf{w}}_{+}, \mathbf{u}_{+}\right)$corresponding to the state $(\mathbf{w}, \mathbf{u})$ can be characterized by the following set of constraints:

$$
\begin{aligned}
w^{j+1} & \leq w_{+}^{j} \leq w^{j} \quad & \text { for } j \in\{1, \ldots, k\} \\
u^{j} & \geq u_{+}^{j} \geq u^{j+1} \quad & \text { for } j \in\{k+1, k+2, \ldots, J-1\} \\
0 & \geq \zeta \geq u^{k+1}, &
\end{aligned}
$$

where we let $w^{k+1}=0$, and $u^{J}=-\infty$.

The sales decision is made after demand is realized. Let $d$ denote the realized demand. Given the current state $(\mathbf{w}, \mathbf{u})$ and the order placement action $\left(\zeta, \hat{\mathbf{w}}_{+}, \mathbf{u}_{+}\right)$in the current period, the sales quantity can be deduced from the state of the next period. Let $w_{+}^{0}$ denote the first component of the state at the end of the current period after sales have occurred; then, the sales quantity is $w^{0}-w_{+}^{0}$. The set of feasible values for $w_{+}^{0}$ is given by

$$
w^{0}-d \leq w_{+}^{0} \quad \text { and } \quad w^{1} \leq w_{+}^{0} \leq w^{0}
$$


since the sales quantity is non-negative and can neither exceed demand nor the inventory at stage 0 , that is, $w^{0}-w^{1}$.

We now comment on the transition of states from one period to the next. Given the bi-echelon state vector $(\mathbf{w}, \mathbf{u})$ and the action vector $\left(\zeta, \hat{\mathbf{w}}_{+}, \mathbf{u}_{+}, w_{+}^{0}\right)$, the order quantity $-\zeta$ at stage $k$ shifts the corresponding amount of inventory from stage $k+1$ to stage $k$. Thus, in the next period, each $w^{j}$ increases by $-\zeta$ for $j \in\{0,1, \ldots, k\}$ and each $u^{j}$ also increases by $-\zeta$ for $j \in\{k+1, k+2, \ldots, J-1\}$. (See the definitions of $w^{j}$ and $u^{j}$ in (2) and (3).) Let $\mathbf{w}_{+}=\left(w_{+}^{0}, \ldots, w_{+}^{k}\right)=\left(w_{+}^{0}, \hat{\mathbf{w}}_{+}\right)$. Then, the state vector in the next period is given by $\left(\mathbf{w}_{+}, \mathbf{u}_{+}\right)-\zeta \mathbf{e}$.

\section{$3.3 \quad L^{\natural}$-Convexity Properties}

Let us now formally define the $T$-period stochastic dynamic program for our system using the state vector $(\mathbf{w}, \mathbf{u})$ and the actions $\left(\zeta, \hat{\mathbf{w}}_{+}, \mathbf{u}_{+}, w_{+}^{0}\right)$. For any $(\mathbf{w}, \mathbf{u})$, let $f_{T+1}(\mathbf{w}, \mathbf{u})=0$. Fix $t \in\{1,2, \ldots, T\}$. Define, for every $\left(\mathbf{w}, \mathbf{u}, \zeta, \mathbf{w}_{+}, \mathbf{u}_{+}\right)$and $d \geq 0$ satisfying constraints $(4)-(7)$,

$$
\begin{aligned}
& \psi_{t}\left(\mathbf{w}, \mathbf{u}, \zeta, \mathbf{w}_{+}, \mathbf{u}_{+} \mid d\right) \\
& =H^{0} \cdot\left(w_{+}^{0}-w^{1}\right)+\sum_{j=1}^{k} H^{j} \cdot\left(w^{j}-w^{j+1}\right)+\sum_{j=k+1}^{J-1} H^{j} \cdot\left(-u^{j}+u^{j-1}\right) \\
& \quad+p \cdot\left(w_{+}^{0}-w^{0}+d\right)+\gamma \cdot f_{t+1}\left(\left(\mathbf{w}_{+}, \mathbf{u}_{+}\right)-\zeta \mathbf{e}\right),
\end{aligned}
$$

where $w^{k+1}=0$ and $u^{k}=0$. (The equation above can be understood by observing that the inventory on hand at stage 0 at the end of the period is $w_{+}^{0}-w^{1}$; the inventory on hand at stage $j$ is $\left(w^{j}-w^{j+1}\right)$ if $j \in\{1, \ldots, k\}$ and $\left(-u^{j}+u^{j-1}\right)$ if $j \in\{k+1, k+2, \ldots, J-1\}$; and the amount of sales lost is $d-\left(w^{0}-w_{+}^{0}\right)$ if $d$ is the demand.)

Now, recall that $\hat{\mathbf{w}}_{+}=\left(w_{+}^{1}, \ldots, w_{+}^{k}\right)$ and $\mathbf{w}_{+}=\left(w_{+}^{0}, w_{+}^{1}, \ldots, w_{+}^{k}\right)$. Let $\hat{\psi}_{t}\left(\mathbf{w}, \mathbf{u}, \zeta, \hat{\mathbf{w}}_{+}, \mathbf{u}_{+} \mid d\right)$ be the minimum value of $\psi_{t}$ optimized over the sales decision $w_{+}^{0}$, i.e.,

$$
\hat{\psi}_{t}\left(\mathbf{w}, \mathbf{u}, \zeta, \hat{\mathbf{w}}_{+}, \mathbf{u}_{+} \mid d\right)=\min _{w_{0}^{+}} \psi_{t}\left(\mathbf{w}, \mathbf{u}, \zeta, \mathbf{w}_{+}, \mathbf{u}_{+} \mid d\right) \text { subject to }(7)
$$


Also, let

$$
\begin{aligned}
\phi_{t}\left(\mathbf{w}, \mathbf{u}, \zeta, \hat{\mathbf{w}}_{+}, \mathbf{u}_{+}\right) & =E_{D_{t}}\left[\hat{\psi}_{t}\left(\mathbf{w}, \mathbf{u}, \zeta, \hat{\mathbf{w}}_{+}, \mathbf{u}_{+} \mid D_{t}\right)\right] \\
g_{t}(\mathbf{w}, \mathbf{u}, \zeta) & =\min _{\hat{\mathbf{w}}^{+}, \mathbf{u}^{+}} \phi_{t}\left(\mathbf{w}, \mathbf{u}, \zeta, \hat{\mathbf{w}}_{+}, \mathbf{u}_{+}\right) \text {subject to }(4) \text { and }(5), \\
\text { and } f_{t}(\mathbf{w}, \mathbf{u}) & =\min _{\zeta} g_{t}(\mathbf{w}, \mathbf{u}, \zeta) \text { subject to }(6) .
\end{aligned}
$$

Theorem 3. For each $t \in\{1, \ldots, T\}$, the functions $g_{t}(\mathbf{w}, \mathbf{u}, \zeta)$ and $f_{t}(\mathbf{w}, \mathbf{u})$ are $L^{\natural}$-convex.

Proof. Note that $f_{T+1}=0$ is $L^{\natural}$-convex. We assume, as an inductive hypothesis, that $f_{t+1}$ is $L^{\natural}$-convex. We will first prove that $\psi_{t}$ is $L^{\natural}$-convex. In the definition of $\psi_{t}$, each of the first four terms is separable and linear, and is therefore $L^{\natural}$-convex. In the last term, since $f_{t+1}$ is $L^{\natural}$-convex, Lemma 2 implies that $f_{t+1}\left(\left(\mathbf{w}_{+}, \mathbf{u}_{+}\right)-\zeta \mathbf{e}\right)$ is $L^{\natural}$-convex in $\left(\mathbf{w}_{+}, \mathbf{u}_{+}, \zeta\right)$. Therefore, $\psi_{t}\left(\mathbf{w}, \mathbf{u}, \zeta, \mathbf{w}_{+}, \mathbf{u}_{+} \mid d\right)$ is a sum of $L^{\natural}$-convex functions and is, therefore, $L^{\natural}$-convex in $\left(\mathbf{w}, \mathbf{u}, \zeta, \mathbf{w}_{+}, \mathbf{u}_{+}\right)$.

Since $\psi_{t}\left(\mathbf{w}, \mathbf{u}, \zeta, \mathbf{w}_{+}, \mathbf{u}_{+} \mid d\right)$ is $L^{\natural}$-convex, Lemma $2(\mathrm{~b})$ implies that $\hat{\psi}_{t}\left(\mathbf{w}, \mathbf{u}, \zeta, \hat{\mathbf{w}}_{+}, \mathbf{u}_{+} \mid d\right)$ is $L^{\natural}$-convex for each $d$. Since the expectation operator preserves $L^{\natural}$-convexity, it follows that $\phi_{t}\left(\mathbf{w}, \mathbf{u}, \zeta, \hat{\mathbf{w}}_{+}, \mathbf{u}_{+}\right)$is $L^{\natural}$-convex. Again by Lemma $2(\mathrm{~b})$, we obtain that both $g_{t}(\mathbf{w}, \mathbf{u}, \zeta)$ and $f_{t}(\mathbf{w}, \mathbf{u})$ are $L^{\natural}$-convex, thus completing the induction.

\subsection{Implications of $L^{\natural}$-Convexity}

Let $\zeta_{t}^{*}(\mathbf{w}, \mathbf{u})$ denote the largest minimizer in the definition of $f_{t}$ for some $t$. Let $q_{t}^{k *}(\mathbf{w}, \mathbf{u})=$ $-\zeta_{t}^{*}(\mathbf{w}, \mathbf{u})$; thus, $q_{t}^{k *}(\mathbf{w}, \mathbf{u})$ is an optimal order quantity for stage $k$. Assuming that $q_{t}^{k *}(\mathbf{w}, \mathbf{u})$ is differentiable, the following inequalities are implied by Lemma $2(\mathrm{c})$ :

$$
\begin{array}{rlrl}
\frac{\partial q_{t}^{k *}}{\partial w^{j}} & \leq 0 & & \text { for } j \in\{0, \ldots, k\} . \\
-\frac{\partial q_{t}^{k *}}{\partial u^{j}} & \geq 0 & & \text { for } j \in\{k+1, \ldots, J-1\} \text { if } k \leq J-2 . \\
\sum_{j=0}^{k} \frac{\partial q_{t}^{k *}}{\partial w^{j}}+\sum_{j=k+1}^{J-1} \frac{\partial q_{t}^{k *}}{\partial u^{j}} & \geq-1, &
\end{array}
$$

where the second summation in the last inequality above does not exist if $k=J-1$.

Notice that Theorem 1 is stated in terms of $\tilde{\mathbf{q}}_{t}^{k *}$, which is a function of $\mathbf{x}$, whereas the inequalities above are stated in terms of $\mathbf{q}_{t}^{k *}$ which is a function of $(\mathbf{w}, \mathbf{u})$. Since $\tilde{\mathbf{q}}_{t}^{k *}$ has not 
been uniquely defined yet, we define

$$
\tilde{\mathbf{q}}_{t}^{k *}(\mathbf{x})=\mathbf{q}_{t}^{k *}(\mathbf{w}, \mathbf{u})
$$

where $\mathbf{w}$ and $\mathbf{u}$ depend on $\mathbf{x}$ through definitions (2) and (3). The results of the theorem follow directly by applying the chain rule for differentiation and the inequalities in (8)-(10).

The case in which $\tilde{\mathbf{q}}_{t}^{k *}(\mathbf{x})$ is not differentiable corresponds to the case in which $\mathbf{q}_{t}^{k *}$ is not differentiable. The details of the proof in this case are provided in the appendix.

\section{Remarks}

In this section, we remark on how Theorem 1 and our analysis extend to other settings.

(i) Arbitrary Lead Times: If the lead times between two successive stages are arbitrary integers, then we can insert a sufficient number of dummy stages between these two stages to ensure that the lead time between any two consecutive stages (including the dummy stages) is one period. We then make sure that no inventory is held at a dummy stage $j$ by inserting an additional constraint $w_{+}^{j}=w^{j+1}$ to (4) and $u_{+}^{j-1}=u^{j}$ to (5). Otherwise, the analysis remains the same and the conclusions of Theorem 1 hold.

(ii) Ordering Capacity: If the quantity ordered by stage $j$ is constrained above by a capacity limit $C A P^{j}$, then we can add the constraint $w^{j+1}-w_{+}^{j+1} \leq C A P^{j}$ to (4) and the constraint $u^{j}-u_{+}^{j} \leq C A P^{j}$ to (5). The rest of the analysis remains unchanged and the conclusions of Theorem 1 hold.

(iii) Backorder Models: If excess demand is backordered instead of being lost, then the only required change to our analysis is replacing (7) with $w^{0}-d=w_{+}^{0}$. In the backorder system without capacity constraints, the conclusion of Theorem 1 trivially follows from the fact that the optimal policy is an echelon order-up-to policy (Clark and Scarf, 1960). However, when capacity constraints are present, then the structure of the optimal policy is not known, in general, and our result here (Theorem 1) is the first result which partially characterizes the optimal policy for such systems. (Parker and Kapuscinski (2004) and Janakiraman and Muckstadt (2008) derive the optimal policy 
structure for such systems but they require the assumption that the capacity limits at all stages are identical.)

We remark that, in addition to the first two extensions mentioned above, Zipkin (2008) also considers systems in which demand is Markov modulated and systems in which there are multiple demand classes; the extensions of our analysis to these cases are identical to Zipkin's. Moreover, he studies the case of stochastic lead times; our analysis here however does not readily extend to this case.

\section{Acknowledgement}

We sincerely thank the reviewers, the Associate Editor and the Area Editor for their comments. In particular, we are grateful to one of the reviewers who suggested the alternate proof provided in Footnote 1 and appropriate references in discrete convexity.

\section{References}

Clark, A., and H. Scarf. 1960. Optimal Policies for a Multiechelon Inventory Problem. Management Science 6:475-490.

Fujishige, S. 2005. Submodular Functions and Optimization. Second ed, Volume 58 of Annals of Discrete Mathematics. Amsterdam, The Netherlands: Elsevier Science.

Janakiraman, G., and J. Muckstadt. 2008. A Decomposition Approach for a Class of Capacitated Serial Systems. Forthcoming, Operations Research.

Karlin, S., and H. Scarf. 1959. Inventory Models of the Arrow-Harris-Marschak Type with Time Lag. In Studies in the Mathematical Theory of Inventory and Production, ed. J. Arrow, S. Karlin, and H. Scarf, Chapter 9, 155-178.

Lu, Y., and J. Song. 2005. Order-Based Cost Optimization in Assemble-to-Order Systems. Operations Research 53 (1): 151-169.

Morton, T. 1969. Bounds on the Solution of the Lagged Optimal Inventory Equation with no Demand Backlogging and Proportional Costs. SIAM Review 11 (4): 572-596. 
Murota, K. 2003. Discrete Convex Analysis, Volume 10 of SIAM Monographs on Discrete Mathematics and Applications. Philadelphia, PA, USA: Society for Industrial and Applied Mathematics.

Murota, K., and A. Shioura. 2004. Fundamental Properties of M-Convex and L-Convex Functions in Continuous Variables. IEICE Transactions on Fundamentals of Electronics, Communications and Computer Sciences 87 (5): 1042-1052.

Parker, R., and R. Kapuscinski. 2004, Sept.-Oct.. Optimal Policies for a Capacitated TwoEchelon Inventory System. Operations Research 52 (5): 739-755.

Topkis, D. M. 1998. Supermodularity and Complementarity. Princeton, New Jersey: Princeton University Press.

Zipkin, P. 2008. On the Structure of Lost-Sales Inventory Models. Operations Research 56 (4): 937-944. 


\section{A Details for the Proof of Theorem 1: The Case where $\mathbf{q}_{t}^{k *}$ is not Differentiable}

Recall that $f_{t}(\mathbf{w}, \mathbf{u})=\min _{\zeta} g_{t}(\mathbf{w}, \mathbf{u}, \zeta)$ subject to $(6)$, that $\zeta_{t}^{*}(\mathbf{w}, \mathbf{u})$ is the largest minimizer in this definition, and $q_{t}^{k *}(\mathbf{w}, \mathbf{u})=-\zeta_{t}^{*}(\mathbf{w}, \mathbf{u})$. We know from Theorem 3 that $g_{t}(\mathbf{w}, \mathbf{u}, \zeta)$ is $L^{\natural}$-convex. Therefore, Lemma 2 (c) implies that

$$
\begin{aligned}
& q_{t}^{k *}(\mathbf{w}, \mathbf{u}) \text { is nonincreasing in }(\mathbf{w}, \mathbf{u}), \text { and } \\
& q_{t}^{k *}((\mathbf{w}, \mathbf{u})+\epsilon \mathbf{e}) \geq q_{t}^{k *}(\mathbf{w}, \mathbf{u})-\epsilon \text { for } \epsilon>0
\end{aligned}
$$

where $\mathbf{e}=(1,1, \ldots, 1)$ is the vector of 1 's. We will show the inequalities in the statement of Theorem 1 for the non-differentiable case using (11)-(12) along with the definition of the transformation from $\mathbf{x}$ to $(\mathbf{w}, \mathbf{u})$ in $(2)-(3)$. These inequalities are directly implied by the following five statements, which we will prove in the remainder of this section:

$$
\begin{aligned}
\frac{\tilde{q}_{t}^{k *}\left(\mathbf{x}+\boldsymbol{\epsilon}^{0}\right)-\tilde{q}_{t}^{k *}(\mathbf{x})}{\epsilon} & \leq 0 \quad \forall k \in\{0, \ldots, J-1\} \\
\frac{\tilde{q}_{t}^{k *}\left(\mathbf{x}+\boldsymbol{\epsilon}^{J-1}\right)-\tilde{q}_{t}^{k *}(\mathbf{x})}{\epsilon} & \geq 0 \quad \forall k \in\{0, \ldots, J-2\} \\
\left(\frac{\tilde{q}_{t}^{k *}\left(\mathbf{x}+\boldsymbol{\epsilon}^{k+1}\right)-\tilde{q}_{t}^{k *}(\mathbf{x})}{\epsilon}\right)-\left(\frac{\tilde{q}_{t}^{k *}\left(\mathbf{x}+\boldsymbol{\epsilon}^{k}\right)-\tilde{q}_{t}^{k *}(\mathbf{x})}{\epsilon}\right) & \leq 1 \quad \forall k \in\{0, \ldots, J-2\} \\
\left(\frac{\tilde{q}_{t}^{k *}\left(\mathbf{x}+\boldsymbol{\epsilon}^{j}\right)-\tilde{q}_{t}^{k *}(\mathbf{x})}{\epsilon}\right)-\left(\frac{\tilde{q}_{t}^{k *}\left(\mathbf{x}+\boldsymbol{\epsilon}^{j-1}\right)-\tilde{q}_{t}^{k *}(\mathbf{x})}{\epsilon}\right) & \leq 0 \quad \forall(j, k) \in\{1 \leq j \leq k\} . \\
\left(\frac{\tilde{q}_{t}^{k *}\left(\mathbf{x}+\boldsymbol{\epsilon}^{j}\right)-\tilde{q}_{t}^{k *}(\mathbf{x})}{\epsilon}\right)-\left(\frac{\tilde{q}_{t}^{k *}\left(\mathbf{x}+\boldsymbol{\epsilon}^{j-1}\right)-\tilde{q}_{t}^{k *}(\mathbf{x})}{\epsilon}\right) & \geq 0 \quad \forall(j, k) \in\{k+2 \leq j \leq J-1\} .
\end{aligned}
$$

To prove statements (13) and (14), observe that (11) implies

$$
\begin{aligned}
\tilde{q}_{t}^{k *}\left(\mathbf{x}+\boldsymbol{\epsilon}^{0}\right)-\tilde{q}_{t}^{k *}(\mathbf{x}) & =q_{t}^{k *}\left((\mathbf{w}, \mathbf{u})+\boldsymbol{\epsilon}^{0}\right)-q_{t}^{k *}(\mathbf{w}, \mathbf{u}) \leq 0 \quad \text { and } \\
\tilde{q}_{t}^{k *}\left(\mathbf{x}+\boldsymbol{\epsilon}^{J-1}\right)-\tilde{q}_{t}^{k *}(\mathbf{x}) & =q_{t}^{k *}\left((\mathbf{w}, \mathbf{u})-\boldsymbol{\epsilon}^{J-1}\right)-q_{t}^{k *}(\mathbf{w}, \mathbf{u}) \geq 0
\end{aligned}
$$

(Throughout this proof, we let $(\mathbf{w}, \mathbf{u})$ be the transformed vector corresponding to $\mathbf{x}$.) 
We show statement (15) by applying (12) as follows. Then,

$$
\begin{aligned}
& \left(\tilde{q}_{t}^{k *}\left(\mathbf{x}+\boldsymbol{\epsilon}^{k+1}\right)-\tilde{q}_{t}^{k *}(\mathbf{x})\right)-\left(\tilde{q}_{t}^{k *}\left(\mathbf{x}+\boldsymbol{\epsilon}^{k}\right)-\tilde{q}_{t}^{k *}(\mathbf{x})\right) \\
& \quad=\tilde{q}_{t}^{k *}\left(\mathbf{x}+\boldsymbol{\epsilon}^{k+1}\right)-\tilde{q}_{t}^{k *}\left(\mathbf{x}+\boldsymbol{\epsilon}^{k}\right) \\
& \quad=q_{t}^{k *}\left((\mathbf{w}, \mathbf{u})-\boldsymbol{\epsilon}^{k+1}-\cdots-\boldsymbol{\epsilon}^{J-1}\right)-q_{t}^{k *}\left((\mathbf{w}, \mathbf{u})+\boldsymbol{\epsilon}^{0}+\cdots+\boldsymbol{\epsilon}^{k}\right) \\
& \leq \epsilon
\end{aligned}
$$

where the second equality follows from (2)-(3), and the inequality follows from (12). This completes the proof of statement (15).

Statement (16) follows from

$$
\begin{aligned}
& \left(\tilde{q}_{t}^{k *}\left(\mathbf{x}+\boldsymbol{\epsilon}^{j}\right)-\tilde{q}_{t}^{k *}(\mathbf{x})\right)-\left(\tilde{q}_{t}^{k *}\left(\mathbf{x}+\boldsymbol{\epsilon}^{j-1}\right)-\tilde{q}_{t}^{k *}(\mathbf{x})\right) \\
& \quad=q_{t}^{k *}\left((\mathbf{w}, \mathbf{u})+\boldsymbol{\epsilon}^{0}+\cdots+\boldsymbol{\epsilon}^{j-1}+\boldsymbol{\epsilon}^{j}\right)-q_{t}^{k *}\left((\mathbf{w}, \mathbf{u})+\boldsymbol{\epsilon}^{0}+\cdots+\boldsymbol{\epsilon}^{j-1}\right) \\
& \quad \leq 0
\end{aligned}
$$

where the inequality follows from (11). The proof of statement (17) is similar. 


\section{Response to the Reviewers}

We sincerely thank the reviewers, the Associate Editor and the Area Editor for their comments. In particular, we are grateful to the reviewer who suggested an alternate proof and several references on discrete convexity. We have addressed all the comments and have marked the corresponding changes in blue font. 